\title{
Emergence of Planck's Constant from Iterated Maps
}

\author{
Ervin Goldfain*
}

Advanced Technology and Sensor Group, Welch Allyn Inc., Skaneateles Falls, NY 13153

\begin{abstract}
Iterations of continuous maps are the simplest models of generic dynamical systems. In particular, circle maps display several key properties of complex dynamics, such as phaselocking and the quasi-periodicity route to chaos. Our work points out that Planck's constant may be derived from the scaling behavior of circle maps in the asymptotic limit.
\end{abstract}

Keywords: Action quantization, Planck's constant, iterated maps, circle maps, winding numbers.

\section{Introduction}

In is known that the probability amplitude for a quantum particle to start at the spacetime point $\left(x_{a}, t_{a}\right)$ and end up at $\left(x_{b}, t_{b}\right)$ is the sum of contributions $\varphi[x(t)]$ from each path, as described by

$$
\bar{K}(b, a)=\sum_{p a t h s} \varphi[x(t)]
$$

Every path in (1) contributes with a phase proportional to the classical action $S$, namely

$$
\varphi[x(t)]=\text { const. } \exp \{(i / \hbar) S[x(t)]\}
$$

The classical approximation of (2) corresponds to the case $S>>\hbar$, with the phase $S / \hbar$ being a large angle. Shifting a given path by a small amount $\delta x$ leads to a negligible change of the action on the classical scale, yet it produces significant changes at a scale commensurate with $\hbar$. At this scale, the exponential function in (2) undergoes fast fluctuations in sign and the overall contribution to (1) cancels out to zero. Neighboring paths in the proximity of the classical path $\bar{x}(t)$ reinforce each other by constructive interference. This is the path that minimizes the action functional ( $\delta S=0$ ), a condition consistent with $\hbar=0$.

\footnotetext{
*Correspondence: Ervin Goldfain, Ph.D., Photonics CoE, Welch Allyn Inc., Skaneateles Falls, NY 13153, USA E-mail: ervingoldfain@gmail.com
} 
In the standard formulation of Quantum Mechanics, there are no additional fluctuations of the exponential besides the intrinsic oscillations discussed above. It is not unreasonable to assume that, on sufficiently short time scales, background fluctuations start to develop and act upon $S$ as periodic or non-periodic perturbations. The "noise-like" nature of these perturbations follow from the inherent instability brought about by probing phenomena on short time scales.

In light of these considerations, it makes sense to model the dynamics of the action $S$ using the general theory of coupled oscillators. This is the object of the next section.

\section{Circle maps and the perturbed dynamics of classical action}

We assume that the background fluctuations are the "driver" oscillator with period $T(B)$ and frequency $v(B)=T^{-1}(B)$. Likewise, let the intrinsic fluctuations of $S$ arising from (2) be characterized by the period $T(S)$ and frequency $v(S)=T^{-1}(S)$. If the background and action fluctuations are uncoupled, the successive points of the Poincaré map are given by [1]

$$
\begin{gathered}
S_{n}=S(t=n T(B)) \\
S_{n+1}-S_{n}=2 \pi \Omega
\end{gathered}
$$

or

$$
S_{n+1}=S_{n}+2 \pi \Omega
$$

in which the "bare" winding number is the ratio

$$
\Omega=\frac{T(S)}{T(B)}=\frac{v(B)}{v(S)}
$$

When there is coupling between background and action fluctuations, the map (5) becomes the sine circle map [1-2]

$$
S_{n+1}=S_{n}+2 \pi \Omega-K \sin \left(S_{n}\right)
$$

which can be alternatively presented as

$$
\theta_{n+1}=\theta_{n}+\Omega-\left(\frac{K}{2 \pi}\right) \sin \left(2 \pi \theta_{n}\right)
$$

Here, $n \in \mathbf{N}$ is the iteration index, $K$ denotes the coupling strength and 
Prespacetime Journal| July 2020| Volume 11 | Issue 4 | pp. 330-333

$$
S_{n}=2 \pi \theta_{n}
$$

The winding number of the map (7) or (8) is defined as

$$
W(K)=\lim _{n \rightarrow \infty} \frac{\theta_{n}-\theta_{0}}{n}=\lim _{n \rightarrow \infty} \frac{S_{n}-S_{0}}{2 \pi n}
$$

and characterizes the average number of $2 \pi$ rotations per iteration [2].

It is important to note that (6) is different from (10) when $K \neq 0$. By contrast, $W(K=0)=\Omega$ when there is no coupling between background and action fluctuations, in which case $W(0)$ denotes the angular increment at each iteration step $n$.

Two situations of interest exist concerning the behavior of (10) when $0<K<1$, namely [1-2]:

a) When (10) is a rational number, the motion corresponds to stable cycles in a finite range of bare winding numbers $0 \leq \Omega \leq 1$,

b) Stable quasi-periodic orbits develop when (10) is an irrational number and the motion occurs on a Cantor set. Moreover, if the quasi-periodic orbits have winding numbers (10) that reside on the Cantor set at $K=1$, these orbits necessarily transition to chaos for $K>1$.

Analysis shows that the transition to chaos in either (7) or (8) relates to a sequence of winding numbers $\left\{W_{n}\right\}$ that converges geometrically according to

$$
W_{n}=W_{\infty}-c \bar{\delta}^{-n}=\bar{W}-c \bar{\delta}^{-n}
$$

where the convergence rate is determined by the golden mean $\bar{W}$ via

$$
\delta=-\bar{W}^{-2}, \bar{W}=\frac{\sqrt{5}-1}{2}
$$

A similar scaling behavior holds for the sequence of bare winding numbers $\left\{\Omega_{n}\right\}$ and one finds, for a given $|K|<1$,

$$
\lim _{n \rightarrow \infty} \Omega_{n}(K)=\Omega_{\infty}=\bar{W}
$$




\section{Emergence of action quantization: two scenarios}

3.1 ) Consider first the case where $1<<n<\infty$ and the coupling strength is nearly-vanishing $|K|<<1$. By (7) and (13) we obtain the first interpretation of action quantization in the form

$$
\delta S_{n+1, n}=S_{n+1}-S_{n}=2 \pi \bar{W}
$$

Note that the condition $|K|<<1$ is consistent with the separation of the high and low energy scales in effective Quantum Field Theory [3].

3.2) Next, consider again the case $1<<n<\infty$ and refer to (10)-(12). The second interpretation of action quantization assumes the form

$$
\delta S_{n, 0}=S_{n}-S_{0}=n(2 \pi \bar{W})
$$

In closing, we bring up a couple of observations:

a) It is apparent from (6) that $\Omega \rightarrow 0$ when $T(B)>>T(S)$. In this case, the background perturbation becomes insignificant and one is led to the action invariance of classical mechanics $\left(S_{n+1}=S_{n}\right)$.

b) According to (14) and (15), Planck's constant emerges as a fixed number related to the golden-mean. The precise numerical value of this constant is irrelevant as Planck's constant can be set to $\hbar=1$ in natural units.

Received May 31, 2020; Accepted July 26, 2020

\section{References}

1. Jackson E. A., "Perspectives of Nonlinear Dynamics", Cambridge Univ. Press, 1992, vol.1

2. McCauley J. L., "Chaos, Dynamics and Fractals", Cambridge Univ. Press, 1994.

3. Duncan A., "The Conceptual Framework of Quantum Field Theory", Oxford Univ. Press, 2012. 